\title{
Analysis of molecular mechanism for acceleration of polyembryony using gene functional annotation pipeline in Copidosoma floridanum
}

\author{
Takuma Sakamoto ${ }^{1}$, Maaya Nishiko², Hidemasa Bono ${ }^{3}$, Takeru Nakazato ${ }^{3}$, Jin Yoshimura ${ }^{4,5,6}$,
} Hiroko Tabunoki ${ }^{1,2^{*}}$ (D) and Kikuo Iwabuchi ${ }^{2}$

\begin{abstract}
Background: Polyembryony is defined as the formation of several embryos from a single egg. This phenomenon can occur in humans, armadillo, and some endoparasitoid insects. However, the mechanism underlying polyembryogenesis in animals remains to be elucidated. The polyembryonic parasitoid wasp Copidosoma floridanum oviposits its egg into an egg of the host insect; eventually, over 2000 individuals will arise from one egg. Previously, we reported that polyembryogenesis is enhanced when the juvenile hormone $(\mathrm{JH})$ added to the culture medium in the embryo culture. Hence, in the present study, we performed RNA sequencing (RNA-Seq) analysis to investigate the molecular mechanisms controlling polyembryogenesis of $C$. floridanum. Functional annotation of genes is not fully available for C.floridanum; however, whole genome assembly has been archived. Hence, we constructed a pipeline for gene functional annotation in C. floridanum and performed molecular network analysis. We analyzed differentially expressed genes between control and $\mathrm{JH}$-treated molura after $48 \mathrm{~h}$ of culture, then used the tblastx program to assign whole C. floridanum transcripts to human gene.
\end{abstract}

Results: We obtained 11,117 transcripts in the $\mathrm{JH}$ treatment group and identified 217 differentially expressed genes compared with the control group. As a result, $76 \%$ of C. floridanum transcripts were assigned to human genes. Gene enrichment analysis revealed genes associated with platelet degranulation, fatty acid biosynthesis, cell morphogenesis in the differentiation and integrin signaling pathways were fluctuated following $\mathrm{JH}$ treatment. Furthermore, Cytoscape analysis revealed a molecular interaction that was possibly associated with polyembryogenesis .

Conclusions: We have constructed a pipeline for gene functional annotation of C. floridanum, and identified transcripts with high similarity to human genes during early embryo developmental. Additionally, this study reveals new molecular interactions associated with polyembryogenesis; these interactions could indicate the molecular mechanisms underlying polyembryony. Our results highlight the potential utility of molecular interaction analysis in human twins.

Keywords: Polyembryony, Xanthine dehydrogenase/oxidase, Endoparasitoid, Polyembryogenesis, Copidosoma floridanum

\footnotetext{
* Correspondence: h_tabuno@cc.tuat.ac.jp

${ }^{1}$ Department of United Graduate School of Agricultural Science, Tokyo

University of Agriculture and Technology, Tokyo, Japan

2Department of Science of Biological Production, Graduate School of

Agriculture, Tokyo University of Agriculture and Technology, Tokyo, Japan

Full list of author information is available at the end of the article
}

(c) The Author(s). 2020 Open Access This article is distributed under the terms of the Creative Commons Attribution 4.0 International License (http://creativecommons.org/licenses/by/4.0/), which permits unrestricted use, distribution, and reproduction in any medium, provided you give appropriate credit to the original author(s) and the source, provide a link to the Creative Commons license, and indicate if changes were made. The Creative Commons Public Domain Dedication waiver (http://creativecommons.org/publicdomain/zero/1.0/) applies to the data made available in this article, unless otherwise stated. 


\section{Background}

The development of a single-cell egg into a multicellular organism begins with cell cleavage; however, polyembryogenesis-in which many embryos are produced from one egg-occurs in some species. Although identical twins are representative examples of human polyembryogenesis, the incidence of this situation is as low as $0.3 \%$ [1]. Armadillos (Dasypus) are the only mammals to exhibit obligatory polyembryony, developing from one egg to four individuals through embryonic shield separation [2,3]. Owing to the ethical limitations of experiments with animal, the phenomenon of polyembryogenesis remains poorly studied.

Furthermore, the occurrence of polyembryogenesis has been reported for insects such as the endoparasitic wasps Braconidae, Dryinidae, Platygasteridae, and Encyrtidae, and research on developmental patterns has progressed rapidly in recent years [4]. The identification of molecules associated with polyembryogenesis could further our understanding of the mechanisms underlying the regulation of this process in animals.

The endoparasitic wasp Copidosoma floridanum (Hymenoptera: Encyrtidae) is an egg-larval parasitoid of the plusiine moth Thysanoplusia intermixta. The egg developmental stage of $T$. intermixta lasts 4 days, the larval developmental stage around 20 days, and pupal developmental lasts around 8 days under experimental conditions [5] (Fig. 1). In standard experimental conditions, C. floridanum parasitizes the 2-day old egg of $T$. intermixta, ultimately producing nearly 2000 cloned embryos from either a fertilized egg (which develops into females) or an unfertilized egg (which develops into males) (Fig. 1). Although almost all insects exhibit egg segmentation due to superficial cleavage, C. floridanum

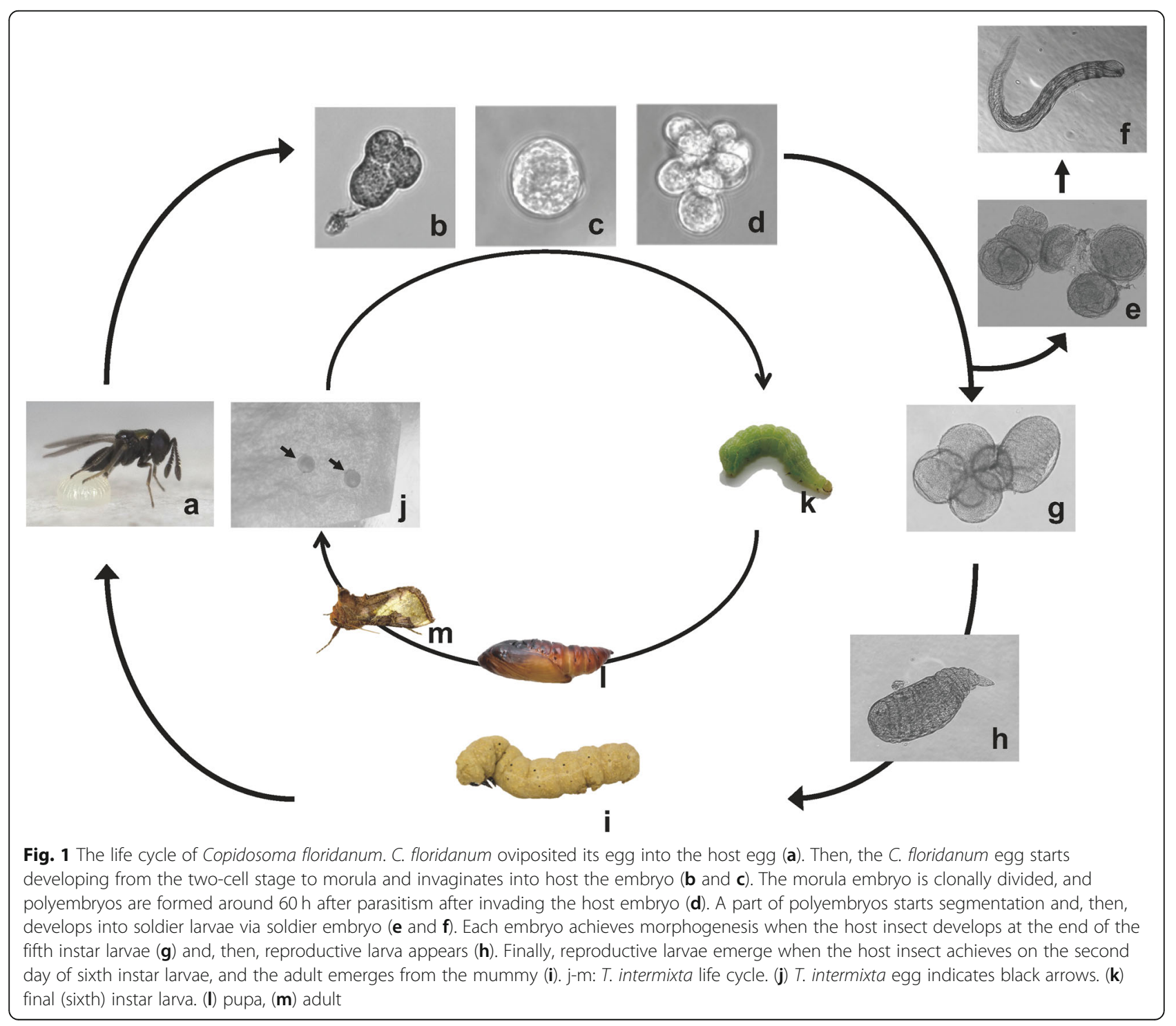


egg segments undergo holoblastic cleavage. In C. floridanum, cell cleavage begins when the egg is laid into a host egg (Fig. 1a). The C. floridanum egg then starts to develop from the two-cell stage to a morula after the $C$. floridanum embryo invades the host embryo (Fig. 1b). The egg of $C$. floridanum comprises an embryonic cell and an anterior cell from a polar body [6]. The anterior cell develops into an extraembryonic syncytium, which forms the outer part of the morula. The extraembryonic syncytium from the anterior cell wraps around the blastomeres after the chorion is removed and morula form (Fig. 1c). The motility of early morulae enable them to invade the host embryo and then develop into polyembryos when morulae movement ceases after entry into the host embryo [7] (Fig. 1d). A subset of polyembryos starts to develop into soldier larvae from embryos without primary germ cells (Fig. 1e and f). Each embryo undergoes morphogenesis when the host insect reaches the end of the final instar of larval development (Fig. 1g). Finally, reproductive larvae emerge when the host insect reaches the second day of the final instar of larval development (Fig. 1h), and adults finally emerge (Fig. 1i) [8, 9]. Although the process by which C. floridanum polyembryony progresses in host embryos is known, the molecular mechanisms remain obscure.

Previously, we reported polyembryogenesis to be accelerated by exposure to juvenile hormone $(\mathrm{JH})$ or the $\mathrm{JH}$ analog methoprene, under culture conditions [10].
Although the molecular function of $\mathrm{JH}$ in the embryogenesis of $C$. floridanum remains unclear, we anticipated that either $\mathrm{JH}$ or methoprene would promote the progress of polyembryogenesis from the two-cell to polymorula stage. Functional annotation of genes is not fully available for C. floridanum; however, wholegenome sequence data is available. Thus, we were unable to analyze the molecular networks in C. floridanum as has been done for Drosophila melanogaster, a model insect. Accordingly, we constructed a pipeline for gene functional annotation of C. floridanum and performed molecular network analysis focusing on gene expression following polyembryony development in response to $\mathrm{JH}$ treatment in the two-cell developmental stage. We also performed RNA sequencing (RNA-Seq) analysis of $C$. floridanum as a model animal for polyembryogenesis to elucidate the molecular mechanisms underlying this process.

\section{Results}

Juvenile hormone accelerates polyembryogenesis

We collected the two-cell-stage embryos and cultured them for 5 days; then, we counted the cultured embryo and evaluated whether these embryos were polymolurae or not (Fig. 2). We found the JH-treated group to exhibit an increased rate of polymolurae compared with the control group. Additionally, polymolurae were identified in the $\mathrm{JH}$-treated group from 2 days after the start of

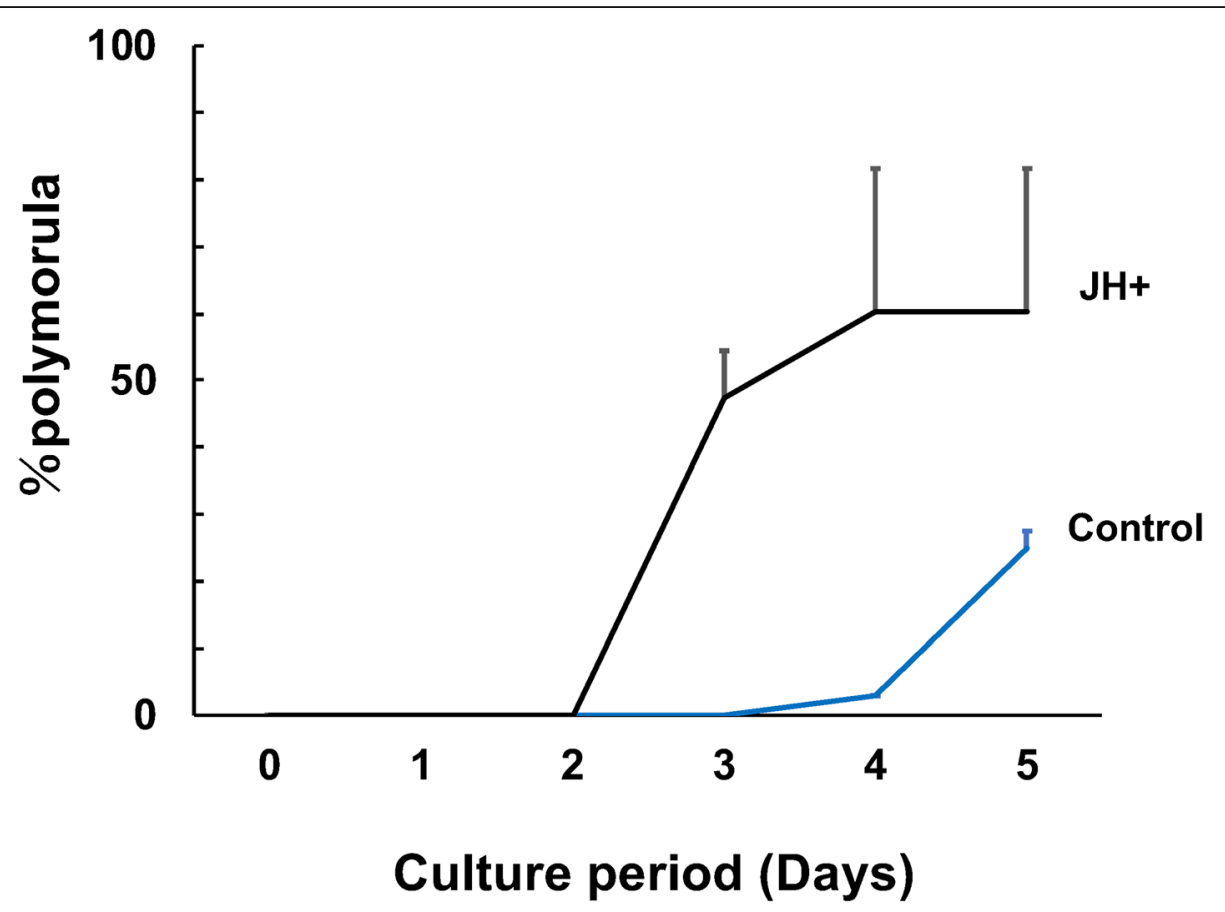

Fig. 2 Polymorula was accelerated by the juvenile hormone $(\mathrm{JH})$ treatment in the culture condition. The early embryo of the two-cell stage was cultured with or without JH. The number of polymorula was counted and plotted on the graph. Notes: Black line, JH-treated group; blue line, control group; vertical axis, rate of polymorula (\%); horizontal axis, culture period (days). Error bars indicate standard deviation (SD) 
culturing, whereas these were only identified in the control group after 4 days after culture. Hence, the development of polymolurae was accelerated by $\mathrm{JH}$ treatment (Fig. 2; Table 1).

\section{Identification of differentially expressed genes and assignment of human homolog}

We constructed a pipeline for functional gene annotation of C. floridanum (Fig. 3). In the present study, we identified 13,160 human homologs of 17,308 total predicted protein dataset in C. floridanum using the current gene functional annotation pipeline. As a result, $C$. floridanum exhibits $76 \%$ gene similarity with humans. Following the results of polymurae analysis, we extracted total RNA for RNA-Seq analysis after $48 \mathrm{~h}$ of cell culture. We performed RNA-Seq analysis on three control samples (SRA accession numbers: DRR138914, DRR138915, and DRR138916) and two JH-treated samples (DRR138917 and DRR138918). The RNA-Seq data were mapped with HISAT2 and StringTie. We obtained 11,117 transcripts from these RNA-Seq data and found that 10,908 of these were commonly expressed in the control and $\mathrm{JH}$-treated groups.

We identified 217 differentially expressed genes (DEGs; false discovery rate [FDR] < 0.05; Fig. 4a). While the expression of 123 of these was increased, 94 were downregulated in the $\mathrm{JH}$-treated group (Fig. 4a, orangecolored dots indicate differentially expressed transcripts).

Next, we assessed the number of $C$. floridanum genes by comparing our transcript dataset with the $C$. floridanum RefSeq datasets. Of the 11,117 transcripts in our $C$. floridanum RNA-Seq datasets, 9417 were present in the C. floridanum RefSeq datasets $(84.7 \%$ coverage). We identified 6098 human homologs out of the 11,117 total transcripts of $C$. floridanum using tblastx with a cutoff $E$-value of $1 \mathrm{e}-10$. Of the $217 \mathrm{DEGs}$ that we identified, 88 corresponded to human genes (Additional file 1: Table S1).

\section{Gene enrichment analysis of differentially expressed genes}

For the gene enrichment analysis using Metascape, we needed to assign human gene IDs to $C$. floridanum genes IDs. Thus, we chose C.floridanum 88 genes, which

Table 1 Impact of polyembryogenesis by the juvenile hormone treatment to the early embryo of Copidosoma floridanum

\begin{tabular}{lll}
\hline Treatment groups & Day of polymorula & Rate of polymorula (\%) \\
\hline Control & $4.88 \pm 0.13$ & 25.0 \\
$\mathrm{JH}$ & $3.16 \pm 0.23$ & 60.3 \\
\hline
\end{tabular}

Control group: experimental 1, $n=10$; experimental $2, n=25$; experimental 3 , $n=21$. Juvenile-hormone-treated group (experimental 1, $n=10$; experimental $2, n=24$; experimental $3, n=23$ ). Polymorula was observed in experimental 1 to 3 groups and the day of polymorula is shown as mean \pm standard deviation. Abbreviations: $J H$ juvenile hormone have sequence similarity to human genes. Of 88 genes that were chosen to compare expression between the two groups, the expression of 42 genes was increased in the $\mathrm{JH}$-treated group, whereas 46 were downregulated following $\mathrm{JH}$ treatment. We imported the list of DEGs and their expression levels into Metascape and converted to their human homologs for gene enrichment analysis. Metascape generated 12 genetic function groups for Gene Ontology (GO), indicating that upregulated genes in the $\mathrm{JH}$-treated group were assigned to platelet degranulation (GO:0002576) and fatty acid biosynthetic process (GO:0006633) (Fig. 4b). Furthermore, Metascape indicated that downregulated genes in the $\mathrm{JH}$-treated group were assigned to cell morphogenesis was involved in differentiation (GO: 0000904) and ST Integrin Signaling Pathway (M3270) (Fig. 4c).

\section{Screening for related molecules using the molecular network analysis by Cytoscape}

We focused on the GO terms platelet degranulation, fatty acid biosynthetic process, and integrin signaling pathway to identify the molecular network that regulates polyembryogenesis. Using the public protein interaction database and Cytoscape, we further explored correlations among the genes involved in these processes. Hence, we identified molecular interactions including filamin-A (FLNA), xanthine dehydrogenase/oxidase $(X D H)$, exportin-1 (XPO1), protein phosphatase slingshot homolog 2 (SSH2), and integrin alpha-4. (ITGA4) as genes that fluctuated in the $\mathrm{JH}$-treated group (Fig. 5a). The Transcripts Per Kilobase Million (TPM) values of these molecules were plotted (Fig. 5b). We found that the mRNA expression of FLNA and $X D H$ increased, while that of SSH2 and ITGA4 decreased following $\mathrm{JH}$ treatment (Fig. 5b). However, the mRNA expression of $\mathrm{XPO1}$ was not by $\mathrm{JH}$ treatment (Fig. 5b).

\section{Discussion}

We investigated gene expression during polyembryogenesis of embryos of $C$. floridanum treated with $\mathrm{JH}$ in the two-cell stage. Additionally, we constructed a pipeline for functional gene annotation of C. floridanum and performed molecular network analysis.

Our previous study revealed that $\mathrm{JH}$ and methoprene accelerate polyembryogenesis; a phenomenon that was also observed when JH I or JH II were added to the culture medium [10]. Methoprene had the strongest effect on promotion of polyembryogenesis, while farnesol, farnesyl acetate, and methyl caproate did not promote polyembryogenesis [10]. Moreover, polyembryogenesis was not promoted by ecdysone (Additional file 2: Figure $\mathrm{S} 1$ ). Hence, only $\mathrm{JH}$ or methoprene promote polyembryogenesis in C. floridanum. 


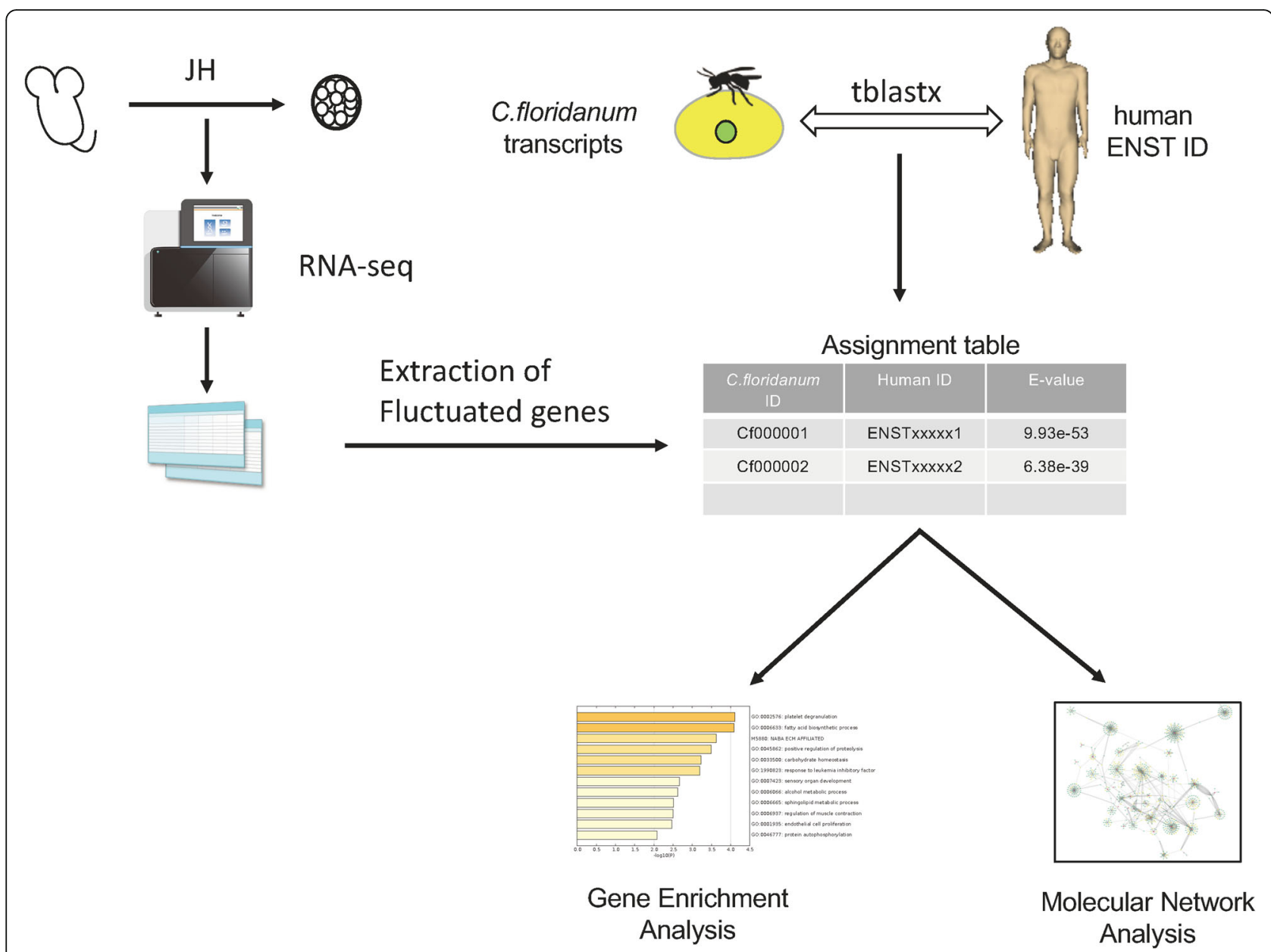

Fig. 3 The strategy of annotation of Copidosoma floridanum transcript to human genes. We performed tblastx between C. floridanum transcripts and human transcripts on public databases to extract fluctuated genes in the juvenile hormone (JH) treatment using the RNA-Seq analysis. Then, we converted transcripts IDs from the C. floridanum RNA-Seq to human Ensembl IDs and constructed assignment table. Finally, we performed the gene enrichment analysis and molecular network analysis using the public database and determined molecular interaction in the polyembryogenesis. The experimental tools, human and machines drawings (https://togotv.dbcls.jp/ja/pics.html) are licensed under Creative Commons Attribution 4.0 International License (CC BY 4.0) (http://creativecommons.org/licenses/by/4.0/deed.en)

The insect hormone $\mathrm{JH}$ is unique in its structure; it has $\alpha$-, and $\beta$-unsaturated methyl ester groups and epoxy groups at both ends of the terpenoid backbone [11]. The importance of $\mathrm{JH}$ in processes such as regulation of molting, pheromone biosynthesis, maturation of gonads, egg development, homeostasis, maintenance of population, and body color change has been reported [12]. Thus, JH is a critical element of insect physiology.

Krüppel homolog $1(K r-h 1)$ is a $\mathrm{JH}$-responsive gene [13]. $K r-h 1$ was not affected by $\mathrm{JH}$ in this study (Additional file 3: Figure S2). Retinoid X receptor (RXR), a type of nuclear receptor that binds to 9-cis retinoic acid [14], may be able to bind to several chemicals that share the structure of retinoic acid [15]. Reportedly, the $\mathrm{JH}$ analog methoprene and methoprene acid can bind to RXR [15], which also functions as a JH receptor in $D$. melanogaster [16]. In this study, the expression of $C$. floridanum RXR-alpha-B tended to be increased following $\mathrm{JH}$ treatment (Additional file 3: Figure S2). Retinoic acid is involved in embryonic development and cell differentiation through its interaction with RXR [17]. This suggests that $\mathrm{JH}$ could act in a similar manner to retinoic acid to affect embryonic development and participate in the retinoic acid signaling pathway in our model of polyembryony.

Additionally, C. floridanum RefSeq datasets were constructed by analyzing male and female adult $C$. floridanum transcriptome. A reference genome sequence of $C$. floridanum from male has been published (https://www. ncbi.nlm.nih.gov/assembly/GCF_000648655.2/). Overall, 17,038 of the estimated proteins have been registered in the NCBI Genome database (https://www.ncbi.nlm.nih. gov/genome/12734?genome_assembly_id=358239). 


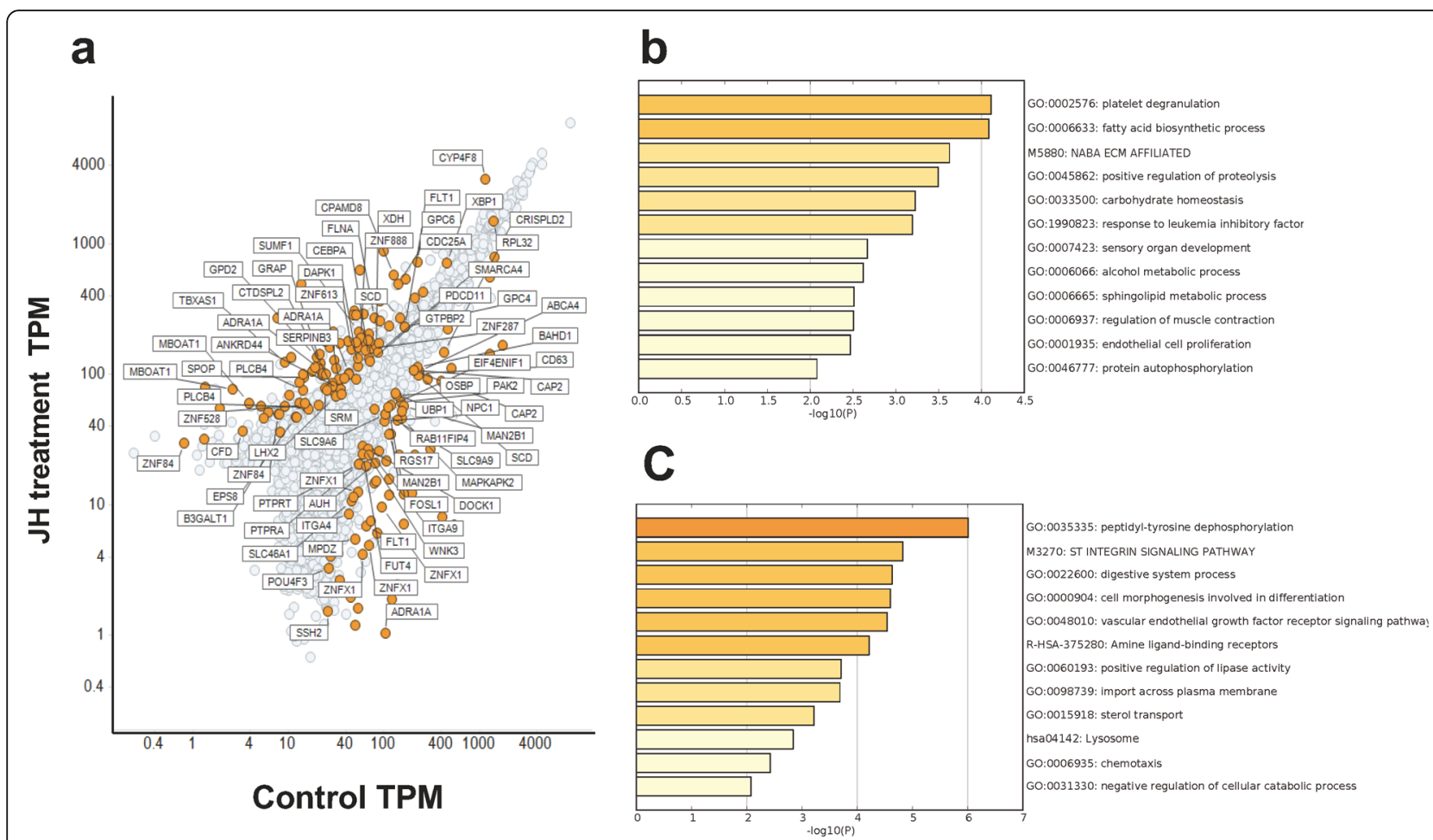

Fig. 4 The extraction of fluctuated transcripts in the juvenile hormone $(\mathrm{JH})$ treatment and gene enrichment analysis. Fluctuated transcripts were extracted and plotted on the graph. Orange dots, transcripts that fluctuated more than two times compared with the control in the $\mathrm{JH}$ treatment (a). The gene enrichment analysis of fluctuated transcripts in the morula using Metascape. A heatmap of enriched terms across the input transcripts lists; different colored bars, $P$ values. (b) $\mathrm{JH}$ upregulated genes; (c) $\mathrm{JH}$ downregulated genes

Previously, we identified human homologs of Bombyx mori and D. melanogaster through systematic BLAST analysis. We found B. mori and D. melanogaster to contain 58 and $63 \%$ of human homologs, respectively [18]. In the present study, we found, $C$. floridanum exhibits $76 \%$ gene similarity with humans. The large number of human homologs that we observed in $C$. floridanum is therefore comparable with these model insects. In the present study, $88 \mathrm{C}$. floridanum genes that showed differential expression when comparing $\mathrm{JH}$ treated and control groups corresponded to human genes, and we input these genes to gene enrichment and molecular network analyses.

Gene enrichment analysis revealed the expression of lipid metabolism-related (GO: 0006633) and platelet degranulation (GO: 0002576) related genes were upregulated in the JH-treated group. The characteristics of genes containing these GO terms have been shown to be involved with vesicle-associated $\mathrm{V}$-soluble $\mathrm{N}$ ethylmaleimide-sensitive factor attachment protein receptor (SNARE) in cellular membrane adhesion [19]. Additionally, genes encoding synaptosomal-associated protein (SNAP) 25 and 29, (SNAPC) 3 and 4, SNAREassociated protein Snapin (SNAPIN), and syntaxin- binding protein $(S T X B P)$ were shown to be commonly expressed in both groups of the present study. Reportedly, these genes are involved in the membrane fusion of neurosecretory cells [20]. Notably, STXBP interacts with these proteins to inactivate membrane fusion [20]. Thus the decreased mRNA expression of STXBP that we observed following $\mathrm{JH}$ treatment may lead to activation of cellular membrane fusion. As the molura promotes fusion with the extraembryonic syncytium, it is possible that $\mathrm{JH}$ treatment could accelerate morula to polyembrogenesis.

The genes SNAP25 and SNAP29, SNAPC3 and SNAPC4, SNAPIN, and STXBP, which we observed to be expressed in C. floridanum morula embryos, also play a role for mediates membrane fusion during exocytosis in the neurosecretory cells of humans [21]. Therefore, $C$. floridanum might employ similar molecular mechanisms for cellular membrane adhesion as human neurosecretory cells.

The morula comprises the outer extraembryonic syncytium and the inner embryonic cell; the extraembryonic syncytium separates by dividing the embryonic cell [22]. Embryonic cells adhere to the extraembryonic syncytium via integrin in the morula. When integrin expression is 


\section{a}

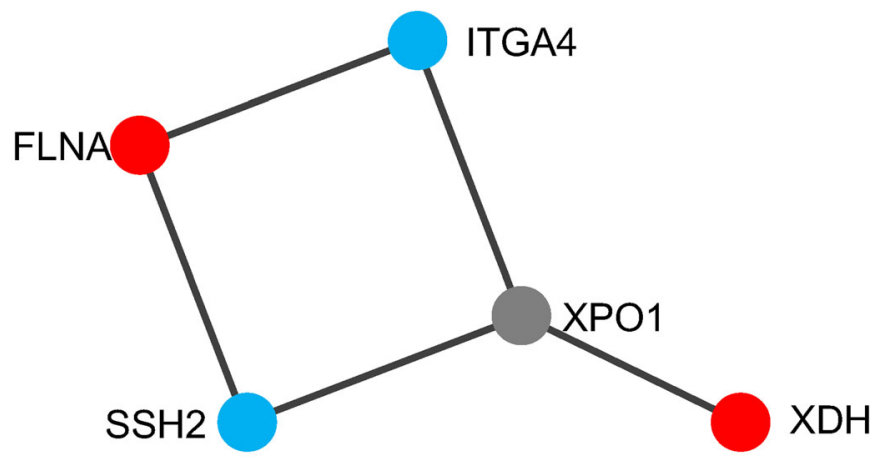

b

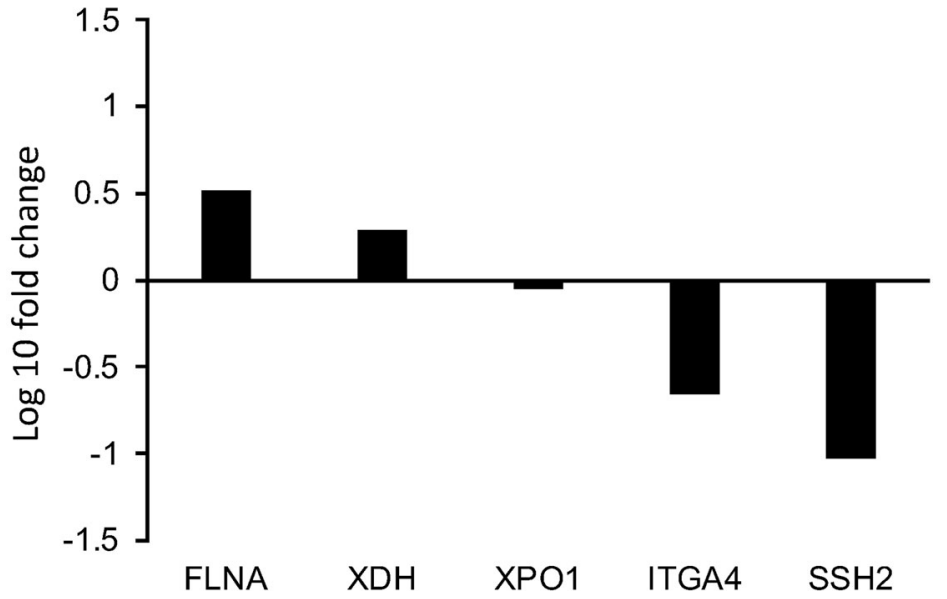

Fig. 5 Screening of the molecular interaction using Cytoscape. The molecular interaction of (a) Cytoscape automatically generates the corresponding molecules as a node on a molecular. Interaction in polyembryogenesis-related genes. A red colored node shows up-regulated genes, blue colored genes shows down-regulated genes, and a gray colored gene is not fluctuation gene. (b) Polyembryogenesis-related genes in juvenile hormone $(\mathrm{JH})$ treatment of molura. The $y$-axis indicates the ratio of the average Transcripts Per Kilobase Million (TPM) values for molura between the control and $\mathrm{JH}$ treatment groups

decreased following exposure to $\mathrm{JH}$, this adhesion may be loose, causing the extraembryonic syncytium to invaginate into the cell gap of embryonic cells. These cells might then divide, resulting in polyembryony. The actin filament crosslinking protein, FLNA, which is present in non-muscle cells [23] might be involved in the division of embryos interacting with $\mathrm{SSH} 2$ and ITGA4. Reportedly, SSH2 mediates actin dynamics [24], while ITGA4 belongs to the integrin family and plays a role in cell adhesion [25]. These molecular interactions might contribute to the polyembryony.

Xanthine dehydrogenase/oxidase is the rate-limiting enzyme of purine metabolism, and a key component in uric acid synthesis. During egg development of $B$. mori the amount of uric acid gradually declines until blastokinesis, after which it increases until egg pigmentation [26]. Remarkably, XHD knockdown has been shown to enhance cell mobility and invasion of HepG2 cells, although no effect on cell proliferation was observed [27]. Furthermore, XDH converts retinoic acid to 9-cis retinal [28], and activity of the enzyme is essential in order for $\mathrm{JH}$ to induce bristle formation and cuticle production on the abdominal epidermis during pupal and adult development [29].

The chemical structures of JH III and retinoic acid are similar, and it is possible that JH III binds to XDH. Then, $\mathrm{XDH}$-bound $\mathrm{JH}$ could enter the nucleus via $X P O 1$, and released $\mathrm{JH}$ might bind to RXR to control subsequent transcription; this molecular correlation could be a novel mechanism triggered by JH. Accordingly, the following model could be proposed for the progression of forming molura to polymolura: To start, embryos are wrapped with extraembryonic syncytium (Fig. 6a). During the formation of polymolurae, the degradation of actin is avoided by reducing $\mathrm{SSH} 2$ expression followed by suppression of ITGA4 expression. As the adhesion of embryonic cells is loose (Fig. 6b), the syncytial membrane facilitates fractionation (Fig. $6 \mathrm{c}$ and d) and promotes polyembryony (Fig. 6e). Hence, XDH plays a key role in embryogenesis via $\mathrm{JH}$, as well as in uric acid synthesis. Overall, the present study reveals novel molecular interactions involved in polyembryogenesis and 


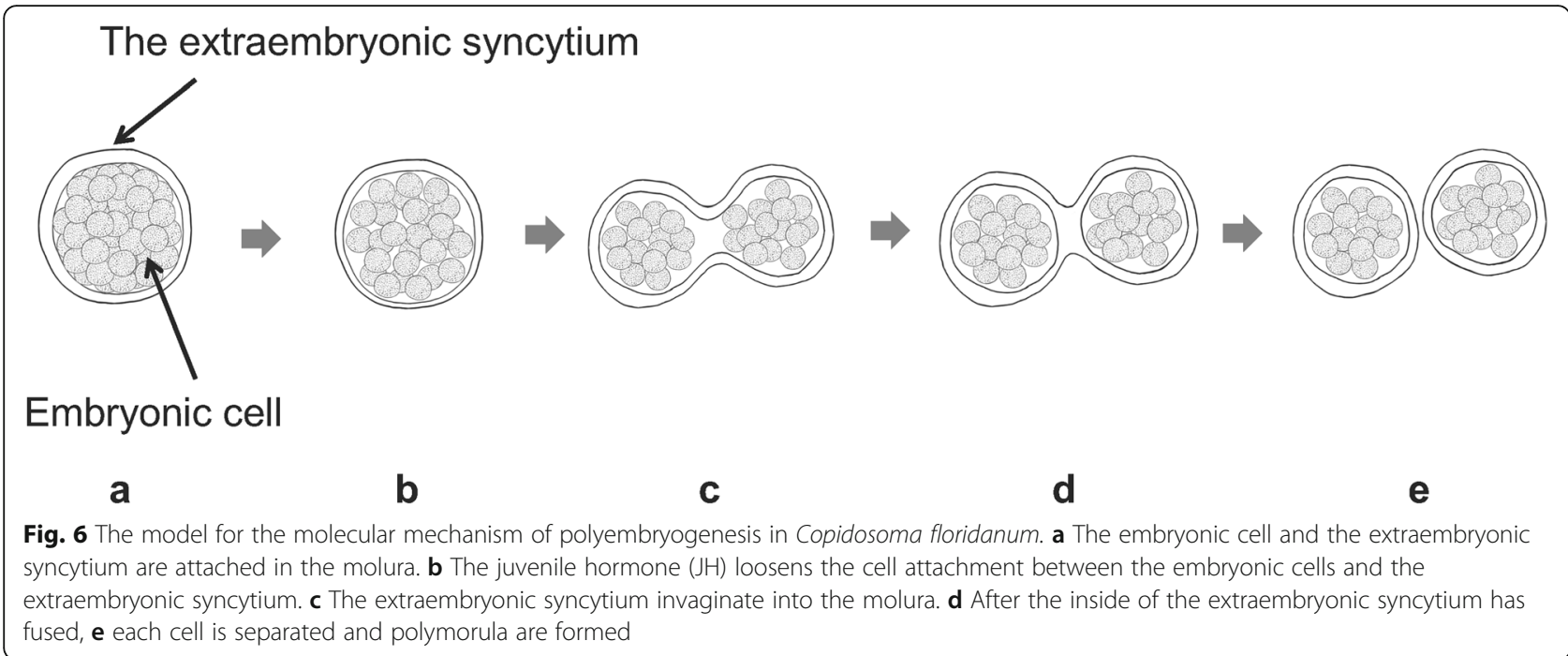

demonstrates the connections that are required for the progression of cell separation in polyembryogenesis.

\section{Conclusions}

In this study, we constructed a pipeline for gene functional annotation of C. floridanum. We identified C. floridanum transcripts with high similarity with several human genes during early embryogenesis, and found that C. floridanum has many more human homologs than $D$. melanogaster. Additionally, we identified new molecular interactions associated with polyembryogenesis, which may enable the elucidatation of the molecular mechanisms underlying polyembryony. Our results highlight the potential utility of molecular interaction analysis for the investigation of polyembryogenesis of humans. In future studies, we intend to investigate the function of these molecules using RNAi in C. floridanum.

\section{Methods}

Insect

We obtained C. floridanum from parasitized T. intermixta larvae from burdock fields in Tokyo, Chiba, and Ibaraki Prefectures. Larvae were maintained with an artificial diet [30] at $25^{\circ} \mathrm{C}$ with a 16 - $\mathrm{h}$ light/8-h dark cycle. T. intermixta adults were fed a $10 \%$ sugar solution absorbed with cotton. C. floridanum adults were fed a $50 \%$ honey solution absorbed with cotton. We used $T$. intermixta eggs within $17 \mathrm{~h}$ post-oviposition for parasitism by $C$. floridanum. The parasitized hosts were kept in the same conditions as non-parasitized hosts.

\section{Embryo culture}

We dissected the two-cell stage of the embryo from $T$. intermixta eggs within 2-h post-oviposition and, then, cultured it with the modified MGM medium [31]. We dissolved JH III (J2000; Sigma-Aldrich Co. Ltd., Munich,
Germany) in ethanol to prepare a stock solution $(10 \mathrm{mg} /$ $\mathrm{mL}$ ); then, $1 \mu \mathrm{L}$ of this stock solution was added to the modified MGM medium as the final concentration was $1 \mu \mathrm{g} / \mathrm{mL}$. In the control group, an equivalent volume of ethanol alone was added to the culture medium. We used two-cell stage embryos produced from $T$. intermixta eggs within $2 \mathrm{~h}$ of oviposition for the control group (experimental 1, $n=10$; experimental 2, $n=25$; experimental $3, n=21$ ) and the $\mathrm{JH}$ group (experimental 1, $n=10$; experimental 2, $n=24$, experimental 3, $n=23$ ).

\section{RNA-Seq analysis}

We isolated total RNA from morulae cultured for 2 days in control (experimental $1, n=24$; experimental $2, n=16$; experimental $3, n=11$ ), and $\mathrm{JH}$ conditions (experimental 1, $n=17$; experimental 2, $n=28$; experimental 3, $n=23$ ) using a combination of TRIzol ${ }^{\circ}$ LS Reagent and the PureLink ${ }^{\circ}$ RNA Extraction Kit (Thermo Fisher Scientific Inc., Valencia, CA) per the manufacturer's instructions. Then, we used an Agilent TapeStation 2200 (Agilent Technologies, Santa Clara, CA) to assess the RNA quality. Additionally, single-end sequencing cDNA libraries were constructed with $100 \mathrm{ng}$ of total RNA from these samples (control group: $n=3$; JH group: $n=3$ ) with a TruSeq ${ }^{\circ}$ Stranded mRNA Sample Preparation Kit (Illumina Inc., San Diego, CA) per the manufacturer's instructions. Next, libraries were sequenced ( $75 \mathrm{bp}$, single-end) on the Illumina NextSeq500 platform, and FASTQ files were assessed by Trim Galore! v0.4.5 (https://www.bioinformat ics.babraham.ac.uk/projects/trim_galore/). Sequencing of the $\mathrm{JH}$ group of experimental 3 was not possible as the creation of a library for RNA-Seq failed. Thus, we omitted the data of this group. Finally, we analyzed gene expression using three biological replicates in the control group and two biological replicates in the JH group. Notably, the C. floridanum genome (GCF_000648655.2) sequence is 
available in the NCBI Genome database (https://www. ncbi.nlm.nih.gov/genome/annotation_euk/Copidosoma floridanum/101/). The obtained FASTQ sequence files were aligned to the genomic reference sequence by HISAT2 v2.1.0 with default parameters [32]. Next, SAM files were converted to BAM files by Samtools v1.8 [33]. Using StringTie v1.3.4, we estimated the transcript abundance, and the count data were extracted by Subread v1.6.0 [34, 35]. All statistical analyses were performed using R software version 3.4.3 (https://www.r-project.org). To normalize the data and compare the control and JHtreated groups, we used the TCC and DEseq2 packages [36]. We generated a scatter plot using TIBCO Spotfire Desktop v7.6.0 with the "Better World" program license (TIBCO Spot re, Inc., Palo Alto, CA; http://spotfire.tibco. $\mathrm{com} /$ better-world-donation-program/). Furthermore, the sequence data (FASTQ files) were deposited to the DDBJ Sequence Read Archive (accession numbers DRR138914DRR138918).

\section{C. floridanum gene functional annotation pipeline for molecular network analysis}

The transcripts were extracted from the C. floridanum genome using gffread software (https://github.com/gper tea/gffread). To annotate C. floridanum gene, we identified genes homologous to those of human by conducting a systematic BLAST search (tblastx) with a cutoff Evalue of significant similarity at $1 \mathrm{e}-10$ (query: C. floridanum cDNA sequence; database: whole human cDNA sequence set from Ensembl database). Using the generated assignment table, we reconstructed conserved pathways common to $C$. floridanum and humans by projecting $C$. floridanum genes onto the human pathway.

\section{Gene enrichment analysis and pathway analysis}

We performed the gene enrichment analysis using Metascape (http://metascape.org/); a gene list for Metascape analysis was generated from the TCC output. Then, we performed the molecular network analysis using IntAct Molecular Interaction Database (https://www.ebi. ac.uk/intact/) and Cytoscape v3.6.1 (http://www.cytos cape.org). We converted the gene IDs from the C. floridanum RNA-Seq data to human Ensembl IDs, using the assignment table described above and, then, input the list of genes obtained from the RNA-Seq data into Cytoscape to obtain the significant molecular interactions with corresponding $E$ values.

\section{Supplementary information}

Supplementary information accompanies this paper at https://doi.org/10. 1186/s12864-020-6559-3.

Additional file 1: Table S1. C. floridanum genes assign to the human gene. Up-regulated genes and down-regulated genes were assigned to the human gene. These genes were used for gene set enrichment analysis.

Additional file 2: Figure S1. Polymorula was accelerated by the juvenile hormone $(\mathrm{JH})$ treatment in the culture condition. The early embryo of the two-cell stage was cultured with or without $\mathrm{JH}$, or Ecdysone. The number of polymorula was counted and plotted on the graph. Solid line, JH treatment; dashed line, treatment without $\mathrm{JH}$; wide dashed line, treatment Ecdysone, vertical axis, a rate of polymorula (\%); horizontal axis, the culture period (day).

Additional file 3: Figure S2. Expression of Krueppel homolog 1-like genes, and retinoic acid receptor RXR-alpha-B in juvenile hormone $(J H)$ treatment of molura. The $y$-axis indicates the ratio of the average Transcripts Per Kilobase Million (TPM) values for molura between the control and $\mathrm{JH}$ treatment groups.

\section{Abbreviations}

DEG: Differentially expressed genes; GO: Gene ontology; JH: Juvenile hormone; RQ: Relative quantification; $\mathrm{RXR}$ : Retinoid $X$ receptor; SNARE: Sensitive factor attachment protein receptor; TPM: Transcripts per kilobase million

\section{Acknowledgements}

We thank to Dr. Madoka Nakai, Tokyo University of Agriculture and Technology for helpful discussion.

\section{Authors' contributions}

Conceived and designed the experiments: TS, HT, and KI. Performed the experiments: TS and MN. Contributed reagents/materials/analysis tools: JY, Kl, and HT. Analyzed the data: TS, HB, TN and HT. Contributed to the writing of the paper under draft version: TS and HT. All authors discussed the data and helped with manuscript preparation. KI and HT supervised the project. All authors read and approved the final manuscript.

\section{Funding}

This work was supported by JSPS KAKENHI grant number JP15H02483 to Kil, $J Y$ and HT, $15 \mathrm{H} 04420$ to JY, 26257405 to JY, $18 \mathrm{H} 02212$ to HT.

The funders declare that they have no role for the design of the study and collection, analysis, and interpretation of data and in writing the manuscript.

\section{Availability of data and materials}

The RNA-seq reads supporting the conclusions of this article are available in the Sequence Read Archive (SRA) with accession IDs: DRR138914,

DRR138915, and DRR138916; control samples, DRR138917 and

DRR138918;JH-treated samples.

Ethics approval and consent to participate

Not applicable.

Consent for publication

Not applicable.

\section{Competing interests}

The authors declare that they have no competing interests.

\section{Author details}

${ }^{1}$ Department of United Graduate School of Agricultural Science, Tokyo University of Agriculture and Technology, Tokyo, Japan. ${ }^{2}$ Department of Science of Biological Production, Graduate School of Agriculture, Tokyo University of Agriculture and Technology, Tokyo, Japan. ${ }^{3}$ Database Center for Life Science (DBCLS), Joint Support-Center for Data Science Research, Research Organization of Information and Systems (ROIS), Mishima, Shizuoka, Japan. ${ }^{4}$ Department of Mathematical Systems Engineering Shizuoka University, Hamamatsu, Japan. ${ }^{5}$ Department of Environmental and Forest Biology, State University of New York College of Environmental Science and Forestry, Syracuse, New York, USA. ${ }^{6}$ Marine Biosystems Research Center, Chiba University, Kamogawa, Chiba, Japan. 
Received: 25 March 2019 Accepted: 6 February 2020

Published online: 11 February 2020

\section{References}

1. Tong S, Caddy D, Short RV. Use of dizygotic to monozygotic twinning ratio as a measure of fertility. Lancet. 1997;349:843-5.

2. Enders AC. Implantation in the nine-banded armadillo: how does a single blastocyst form four embryos? Placenta. 2002;23:71-85.

3. Loughry WJ, Prodöhl PA, McDonough CM, Avise JC. Polyembryony in armadillos. Am Sci. 1998:86:274-9.

4. Ivanova-Kasas OM. Polyembryony in insects. In: Counce SJ, Waddington $\mathrm{CH}$, editors. Developmental systems, vol. 1. New York: Academic Press; 1972. p. 243-71.

5. Uematsu $\mathrm{H}$. The effective temperature and seasonal prevalence of Thysanoplusia intermixta (WARREN) (Lepidoptera: Noctuidae) Proc. Assoc PI Prot Kyushu. 1978;24:109-12.

6. Baehrecke EH, Strand MR. Embryonic morphology and growth of the polyembryonic parasitoid Copidosoma floridanum (Ashmead) (Hymenoptera : Encyrtidae) international J. Insect Morphol Embryol. 1990;19:165-75.

7. Nakaguchi A, Hiraoka T, Endo Y, Iwabuchi K. Compatible invasion of a phylogenetically distant host embryo by a hymenopteran parasitoid embryo. Cell Tissue Res. 2006:324:167-73.

8. Strand MR. Oviposition behavior and progeny allocation of the polyembryonic wasp Copidosoma floridanum (Hymenoptera: Encyrtidae). J Insect Behav. 1989;2:355-69.

9. Grbic M, Nagy LM, Carroll SB, Strand M. Polyembryonic development: insect pattern formation in a cellularized environment. Development. 1996;122:795-804.

10. Iwabuchi K. Effect of juvenile hormone on the embryogenesis of a polyembryonic wasp, Copidosoma floridanum, in vitro. Vitr Cell Dev Biol Anim J Soc Vitr Biol. 1995;31:803-5.

11. Gilbert LI, Granger NA, Roe RM. The juvenile hormones: historical facts and speculations on future research directions. Insect Biochem Mol Biol. 2000;30:617-44.

12. Truman JW, Riddiford LM. The origins of insect metamorphosis. Nature. 1999:401:447-52.

13. Adam G. The retinoic-like juvenile hormone controls the looping of leftright asymmetric organs in Drosophila. Development. 2003;130:2397-406.

14. Germain P, Germain P, Chambon P, Eichele G, Evans RM, Lazar MA, et al. International Union of Pharmacology. LXIII Retinoid X Receptors Pharmacological Reviews. 2006;58:760-72.

15. Harmon MA, Boehm MF, Heyman RA, Mangelsdorf DJ. Activation of mammalian retinoid $X$ receptors by the insect growth regulator methoprene. Proc Natl Acad Sci. 1995:92:6157-60.

16. Jones $\mathrm{G}$, Jones $\mathrm{D}$. Considerations on the structural evidence of a ligandbinding function of ultraspiracle, an insect homolog of vertebrate RXR. Insect Biochem Mol Biol. 2000;30:671-9.

17. Grenier E, Levy E, Tremblay E, Delvin E, Garofalo C, Sane A, et al. Effect of retinoic acid on cell proliferation and differentiation as well as on lipid synthesis, lipoprotein secretion, and apolipoprotein biogenesis. Am J Physiol Liver Physiol. 2007;293:G1178-89.

18. Tabunoki H, Bono H, Ito K, Yokoyama T. Can the silkworm (Bombyx mori) be used as a human disease model? Drug Discov Ther. 2016;10:3-8.

19. Chen X, Tomchick DR, Kovrigin E, Araç D, Machius M, Sü TC. Threedimensional structure of the Complexin/SNARE complex energy of SNARE complex formation may be used to overcome the energy barrier required to bring the synaptic vesicle and plasma membranes together, perhaps. Neuron. 2002;33:397-409.

20. Mohrmann $R$, De Wit $H$, Verhage $M$, Neher $E$, Sørensen JB. Fast vesicle fusion in living cells requires at least three SNARE complexes. Science. 2010; 330:502-5.

21. Südhof TC. Neurotransmitter release: the last millisecond in the life of a synaptic vesicle. Neuron. 2013;80:675-90.

22. Grbić M, Nagy LM, Strand MR. Development of polyembryonic insects: A major departure from typical insect embryogenesis. Dev Genes Evol. 1998; 208:69-81.

23. Nakamura F, Stossel TP, Hartwig JH. The filamins: organizers of cell structure and function. Cell Adhes Migr. 2011;5:160-9.

24. Niwa R, Nagata-Ohashi K, Takeichi M, Mizuno K, Uemura T. Control of actin reorganization by slingshot, a family of phosphatases that dephosphorylate ADF/cofilin. Cell. 2002;108:233-46.
25. Yang JT, Rayburn H, Hynes RO. Cell adhesion events mediated by alpha 4 integrins are essential in placental and cardiac development. Development. 1995; 121:549-60.

26. Hayashi Y. Studies on the xanthine oxidase system in the silkworm, Bombyx mori L. (III) changes of the uric acid content and xanthine dehydrogenase activity in the silkworm egg during embryonic development. Bull. Sericul. Exp. Stat. 1961;30:427-36.

27. Chen GL, Ye T, Chen HL, Zhao ZY, Tang WQ, Wang LS, et al. Xanthine dehydrogenase downregulation promotes TGF $\beta$ signaling and cancer stem cell-related gene expression in hepatocellular carcinoma. Oncogenesis. 2017:6:e382.

28. Taibi G, Di Gaudio F, Nicotra CMA. Xanthine dehydrogenase processes retinol to retinoic acid in human mammary epithelial cells. J Enzyme Inhib Med Chem. 2008;23:317-27.

29. Zhou X. Riddiford LM: rosy function is required for juvenile hormone effects in Drosophila melanogaster. Genetics. 2008;178:273-81.

30. Kawasaki K, Ikeuchi M, Hidaka T. Laboratory rearing method for Acanthoplusia agnata (Lepidoptera: Noctuidae) without change of artifical diet. Japanese J Appl Entomol Zool. 1987;31:78-80.

31. Iwabuchi K. Early Embronic development of a Polyembryonic wasp, Litomastix maculata ISHII, in vivo and in vitro. Appl Entomol Zool. 1991; 26(4):563-70.

32. Pertea M, Kim D, Pertea GM, Leek JT, Salzberg SL. Transcript-level expression analysis of RNA-seq experiments with HISAT, StringTie and Ballgown. Nat Protoc. 2016;11:1650

33. Wysoker A, Fennell T, Marth G, Abecasis G, Ruan J, Li H, et al. The sequence alignment/map format and SAMtools. Bioinformatics. 2009;25:2078-9.

34. Liao Y, Smyth GK, Shi W. The subread aligner: fast, accurate and scalable read mapping by seed-and-vote. Nucleic Acids Res. 2013;41:e108.

35. Liao Y, Smyth GK, Shi W. featureCounts: an efficient general purpose program for assigning sequence reads to genomic features. Bioinformatics. 2014;30:923-30.

36. Sun J, Nishiyama T, Shimizu K, Kadota K. TCC: an R package for comparing tag count data with robust normalization strategies. BMC Bioinformatics. 2013;14:219.

\section{Publisher's Note}

Springer Nature remains neutral with regard to jurisdictional claims in published maps and institutional affiliations.

Ready to submit your research? Choose BMC and benefit from:

- fast, convenient online submission

- thorough peer review by experienced researchers in your field

- rapid publication on acceptance

- support for research data, including large and complex data types

- gold Open Access which fosters wider collaboration and increased citations

- maximum visibility for your research: over $100 \mathrm{M}$ website views per year

At BMC, research is always in progress.

Learn more biomedcentral.com/submissions 